Tropical Journal of Pharmaceutical Research February 2019; 18 (2): 273-278

ISSN: $1596-5996$ (print); 1596-9827 (electronic)

(C) Pharmacotherapy Group, Faculty of Pharmacy, University of Benin, Benin City, 300001 Nigeria.

\title{
Curcumin ameliorates renal impairment in a diabetic rat model
}

\author{
Peiling Bao ${ }^{1}$, Xiaoyan $\mathrm{Wu}^{1 *}$, Sai $\mathrm{Xie}^{2}$, Tao $\mathrm{Li}^{2}$, Aiqiao Feng ${ }^{2}$ \\ ${ }^{1}$ Department of Nephrology, ZhongNan Hospital of WuHan University, Wuhan city, 430071, Hubei province, China, ${ }^{2}$ Department \\ of Nephrology, The Central Hospital of Xiaogan, 2 Plaza Street, Xiaogan City, 432000, Hubei Province, China
}

${ }^{*}$ For correspondence: Email: wuxiaoyankjdj@163.com; Tel: +86-027-67812888

\begin{abstract}
Purpose: To investigate the molecular mechanisms of action of curcumin in regulating kidney injury in diabetic rats.

Methods: Diabetes was induced in male Wistar rats by intraperitoneal administration of streptozotocin (STZ). The rats were divided into four groups, labelled as follows: blank control, positive control of curcumin-untreated STZ-rats, curcumin-treated STZ-rats $(20 \mathrm{mg} / \mathrm{kg})$, and curcumin-treated STZ-rats (50 $\mathrm{mg} / \mathrm{kg}$ ). After 24 weeks, blood glucose, HbA1c, mean arterial pressure (MAP), heart rates, and body weights were measured. Fasting blood samples were also collected for albumin, lipocalin-2, osteopontin, and kidney-injury-molecule 1 (KIM1) The samples were also evaluated by enzyme linked immunosorbent assay (ELISA). Rat kidneys were isolated for assessment of renal impairment by haematoxylin and eosin staining (H\&E), TUNEL assays, polymerase chain reaction (PCR), and western blotting.

Results: Compared with STZ group, STZ + Cur (50 mg/kg) group significantly decreased blood glucose (284.57 $\pm 4.28 \mathrm{mg} / \mathrm{dL}, p<0.01 \mathrm{vs}$. STZ), HbA1c (5.22 $\pm 0.33 \%, p<0.01 \mathrm{vs}$. STZ), and MAP (76 \pm 2 $\mathrm{mmHg}, p<0.05 \mathrm{vs}$. STZ), heart rate (300 $\pm 6 \mathrm{bpm}, p<0.05 \mathrm{vs}$. STZ), and body weight (356 $\pm 6 \mathrm{~g}, p<$ $0.01 \mathrm{vs}$. STZ) were significantly increased. Kidney protein index was significantly increased, indicating improvement of renal pathological damage. The inflammatory and apoptotic cells were less than that of the STZ group in the renal tissues. The mRNA abundance and relative protein expression levels of Wnt $5 a$ and $\beta$-catenin were also enhanced. Curcumin regulation of the Wnt signal pathway was inhibited by protease inhibitor, XAV-939.

Conclusion: These results demonstrated that curcumin treatment in diabetic rats alleviates renal damage by regulating Wnt signal pathway.
\end{abstract}

Keywords: Curcumin, Renal impairment, Diabetes, Wnt signal pathway

\begin{abstract}
This is an Open Access article that uses a funding model which does not charge readers or their institutions for access and distributed under the terms of the Creative Commons Attribution License (http://creativecommons.org/licenses/by/4.0) and the Budapest Open Access Initiative (http://www.budapestopenaccessinitiative.org/read), which permit unrestricted use, distribution, and reproduction in any medium, provided the original work is properly credited.

Tropical Journal of Pharmaceutical Research is indexed by Science Citation Index (SciSearch), Scopus, International Pharmaceutical Abstract, Chemical Abstracts, Embase, Index Copernicus, EBSCO, African Index Medicus, JournalSeek, Journal Citation Reports/Science Edition, Directory of Open Access Journals (DOAJ), African Journal Online, Bioline International, Open-J-Gate and Pharmacy Abstracts
\end{abstract}

\section{INTRODUCTION}

Renal impairment is one type of chronic diabetic microvascular complication. The pathological molecular mechanism is complex, involving different stimuli and signaling pathways. For example, the renin-angiotensin system (RAS), inflammation and autoimmune responses, oxidative stress, and disorders of glucose metabolism could be involved [1]. 
Hyperglycaemia increases the inflammatory responses in kidney tissues, which leads to a series of damaging pathological changes in the renal cells.[2-4] Reducing local inflammatory responses is crucial for the treatment of diabetic nephropathy.

Curcumin is a chemical component extracted from the rhizome of Zingiberaceae plants and has many pharmacological activities, such as anti-inflammatory, anti-tumor and anti-oxidant activities.[4-6] Curcumin is used for diabetes treatment, and it can improve diabetes symptoms via multiple pathways, such as by inhibiting the inflammatory response and improving diabetic kidney function.[7] Previous studies reported that curcumin treatment for 40 days prevented diabetes by inhibiting p300 and the nuclear factor-kB pathway.[8,9] However, the effect and mechanism of curcumin in kidney damage caused by diabetes remain unclear. This study investigated the effect of curcumin on diabetic nephropathy and its signal pathway mechanism.

\section{EXPERIMENTAL}

\section{Materials}

Male Wistar rats $(250$ - $300 \mathrm{~g})$ were purchased from Shanghai SLAC Laboratory (Shanghai). Curcumin (content > $98 \%$ ), chemical reagents, and protease inhibitor XAV-939 were obtained from Sigma-Aldrich (St. Louis, MO, USA). Wnt $5 a, \beta$-catenin, and TGF- $\beta$ antibodies were from Abcam (Burlingame, CA, USA). HbA1c, creatinine, lipocalin-2, osteopontin, and the kidney-injury-molecule 1 (KIM1) assay kit were purchased from Novus Biologicals (Littleton, CO, USA). The blood glucose meter was obtained from Roche Diagnostics (San Jose, CA, USA).

\section{Animal care and treatment}

The animal experiments were approved by the Animal Ethics Committee of the Central Hospital of Xiaogan (no. CXM-017-026), and were in full compliance with the guidelines of the National Institutes of Health (USA) [10]. The rats were housed at room temperature with a $12 \mathrm{~h} / 12 \mathrm{~h}$ light/dark cycle. After 1 week, the rats were randomly divided into four groups. Ten rats were included in the blank control group, and the rest $(n=30)$ were injected with fresh streptozotocin solution (20 or $50 \mathrm{mg} / \mathrm{kg}$ body weight in $0.1 \mathrm{~mol} / \mathrm{L}$ citrate buffer, $\mathrm{pH} 4.5$ ). The rats were given $5 \%$ glucose solution for further development of the diabetic model. When the blood glucose reached $250-400 \mathrm{mg} / \mathrm{dL}$, rats were considered to have moderate diabetes with hyperglycaemia, and were taken for the experiments. Twenty diabetic rats were treated by oral gavage daily with different doses of curcumin for 17 weeks. During the oral administration, all rats were weighed and their mean arterial pressure (MAP) and heart rate were measured. After 24 weeks of feeding, all rats were sacrificed by spine dislocation after fasting for $12 \mathrm{~h}$. Blood samples were centrifuged at $4{ }^{\circ} \mathrm{C}$ at 3,000 rpm for $10 \mathrm{~min}$ for blood glucose and $\mathrm{HbA1c}$ measurements. Kidney tissues were rapidly isolated and stored at $-80^{\circ} \mathrm{C}$ until further analysed.

\section{Blood pressure and heart rate measurement}

A Softron BP-98A catheter (Tokyo, Japan) was used to measure MAP, at the rat tails. The pulse vibration wave was detected by infrared sensor technology. The signal was transformed to calculate the MAP and heart rate after haemodynamic variables were stabilized.

\section{Biomarker detection}

Serum was collected from the rats via eyeball exophthalmia and was used for lipocalin-2, osteopontin, and KIM1 detection using ELISA assays. Albumin measurement used a competitive assay. All biomarker detections were according to the manufacture's protocols.

\section{Histochemical and terminal deoxynucleotidyl transferase dUTP nick end labelling (TUNEL)}

Tissue samples from the kidneys were collected for haematoxylin-eosin (H\&E) staining and TUNEL assays. H\&E staining identified the morphology and kidney damage, and TUNEL was used for apoptosis detection. All images were taken using an optical microscope (DSX 110; Olympus, Tokyo, Japan).

\section{Quantitative real-time PCR}

The rat renal tissues were isolated and total cellular RNA was extracted by TRIzol reagent (Invitrogen, Carlsbad, CA, USA) according to the manufacturer's instructions. Real-time quantitative RT-PCR was performed using the MiniAmp (Thermo Fisher, Scotts Valley, CA, USA) instrument and PCR was carried out in 96well plates (Table 1). The PCR conditions were as follows: initial denaturation was at $95^{\circ} \mathrm{C}$ for 30 seconds, then 40 cycles, with denaturation at $95^{\circ} \mathrm{C}$ for 5 seconds, and annealing at $58^{\circ} \mathrm{C}$ for 15 seconds. After each cycle, the fluorescent product was measured using a single acquisition mode at $58^{\circ} \mathrm{C}$. Target gene (Wnt $5 a$ and $\beta$ catenin) expressions were normalized. Relative gene expression was calculated using the $2^{-\Delta \Delta C t}$ method. Melting curve analyses and standard 
Table 1: Real-time PCR primers for amplification of mRNA

\begin{tabular}{lcc}
\hline Gene & Forward (5'-3') & Reverse (5'-3') \\
\hline Wnt 5a & GCGAGACGGCCTTCACATAC & ACTCCTTGGCAAAGCGGTAG \\
$\beta$-catenin & AACTAAACAGGAAGGGATGGA & GCACAAGAGCCTCTATACCA \\
$\beta$-actin & CACACCTTCTACAATGAGCTG & GTCTCAAACATGATCTGGGTC \\
\hline
\end{tabular}

curves were performed on the PCR products to confirm correct amplification.

\section{Western blotting}

After electrophoresis, the gels were blotted onto PVDF membranes using a Plate Electrodes instrument (Bio-Rad, Hercules, CA, USA). The protein was boiled in SDS loading buffer for 5 min. Cellular proteins were separated by SDSPAGE (10\%) and transferred to a nitrocellulose membrane. Then, the membrane was incubated for $1.5 \mathrm{~h}$ at room temperature with blocking buffer [1\% skim milk and $0.1 \%$ bovine serum albumin (BSA) in Tris-buffered saline (TTBS)]. Membranes were incubated overnight in blocking buffer with the appropriate primary antibody at $4^{\circ} \mathrm{C}$. The membrane was incubated with the appropriate secondary antibody for $1 \mathrm{~h}$. The immunoreactive protein was visualized with ECL.

\section{Wnt signal blocking test}

STZ rats were divided into three groups. Two groups were injected with $100 \mu \mathrm{L} 2 \mathrm{mg} / \mathrm{mL}$ Wnt inhibitor XAV-939. One of the injection groups was treated with $50 \mathrm{mg} / \mathrm{kg}$ curcumin. After the determined period, the rats were euthanized, renal injury was examined by $\mathrm{H} \& \mathrm{E}$ staining and TUNEL staining; and the activation of Wnt signaling pathway was evaluated by western blotting and PCR.

\section{Statistical analysis}

The data are expressed as mean \pm SD. Statistical comparisons of mean values between groups were performed by analysis of variance with the aid of SPSS software, and $p<0.05$ was considered statistically significant.

\section{RESULTS}

\section{Curcumin alleviated symptoms of diabetic rats.}

STZ was injected to induce diabetes in rats. As shown in Figure $1 A$ and $D$, STZ-treated rats exhibited high glucose (446.33 $\pm 2.75 \mathrm{mg} / \mathrm{dL})$ and $\mathrm{HbA1c}(9.01 \pm 0.26 \%)$ compared with that of the control group. The MAP $(62 \pm 1 \mathrm{mmHg})$, heart rate $(225 \pm 3 \mathrm{bpm})$, and body weight (268 \pm 2 g) were decreased. Curcumin was supplemented in the diets of the treated STZ groups. Low and high doses of curcumin were 20 or $50 \mathrm{mg} / \mathrm{kg}$ of curcumin. The STZ + cur (50 $\mathrm{mg} / \mathrm{kg}$ ) group showed a 1.6-fold and 1.8-fold decrease in blood glucose and HbA1c levels, respectively, compared to that of the untreated STZ rats $(284.57 \pm 4.28 \mathrm{mg} / \mathrm{dL}, 5.22 \pm 0.33 \%, p$ $<0.001$, Figure $1 \mathrm{~A}$ and D). The STZ + cur (50 $\mathrm{mg} / \mathrm{kg}$ ) group had increased MAP, heart rate, and body weight $(76 \pm 2 \mathrm{mmHg}, 300 \pm 6 \mathrm{bpm}$, $356 \pm 6 \mathrm{~g}$, respectively; $p<0.05$, Figure $1 \mathrm{~B}, \mathrm{C}$ and $E)$. In addition, these improvements using high-dose curcumin were better than when using low-dose curcumin treatments.

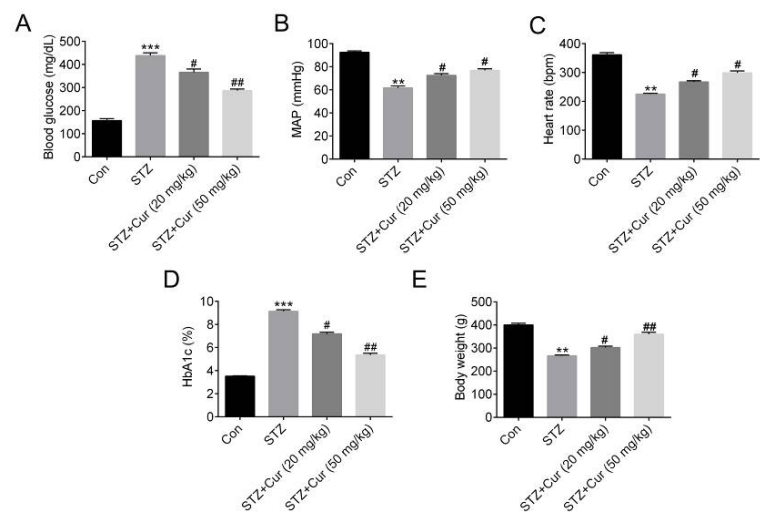

Figure 1: Effect of curcumin (Cur) gavage on diabetic rats. A: Blood glucose; $B$ : MAP; C: Heart rate; D: HbA1c; E: Body weight. ( $n=10$, mean $\pm \mathrm{SD}){ }^{* \star *} p<$ 0.001 STZ vs. control group (Con), ${ }^{\#} p<0.05$, \# $p<$ 0.01: STZ + curcumin vs. STZ. STZ = streptozotocin; $\mathrm{MAP}=$ mean arterial pressure

\section{Curcumin ameliorateded kidney damage in diabetic rats}

As indicated previously, curcumin significantly alleviated the symptoms of diabetes, when using long-term supplementation. To assess whether curcumin could improve kidney damage in an established diabetic model, albumin, lipocalin-2, osteopontin, and KIM1 levels were measured. As shown in Figure 2A-D, the STZ group showed significantly higher levels of albumin, lipocalin-2, osteopontin, and KIM1 than the control group ( $p$ $<0.01)$. After curcumin treatment $(20 \mathrm{mg} / \mathrm{kg})$, all protein levels were significantly decreased $(p<$ 0.05). In addition, Curcumin treatment significantly reduced albumin, lipocalin-2, osteopontin, and KIM1 levels and STZ + Cur (50 $\mathrm{mg} / \mathrm{kg})$ showed even better effects $(p<0.01)$. 
The protein levels of the STZ + Cur $(50 \mathrm{mg} / \mathrm{kg})$ group had a 2 -fold increase compared to that of the STZ group.

Furthermore, to assess whether curcumin improved kidney damage, we used H\&E and TUNEL staining of renal tissues in the four groups (Figure 2E and F). The renal cortex, glomerular glomerulus, and renal tissues showed clear boundaries and had renal tubular dysfunction, glomerular sclerosis and dilation, diffuse mesangial matrix dilatation, and thickening of peripheral vascular wall, indicating that diabetes caused kidney damage; and there were almost no inflammatory cells in the control group. In the STZ group, the glomerular glomerulus was filled with inflammatory cells and the renal cystic wall was thicker than that of the control group. This symptom was ameliorated by curcumin treatment, and the effect was more evident with higher doses. In addition, the apoptotic cells were increased in the STZ group. However, in the STZ + Cur groups decreased numbers of apoptotic cells were observed after high dose curcumin treatment.

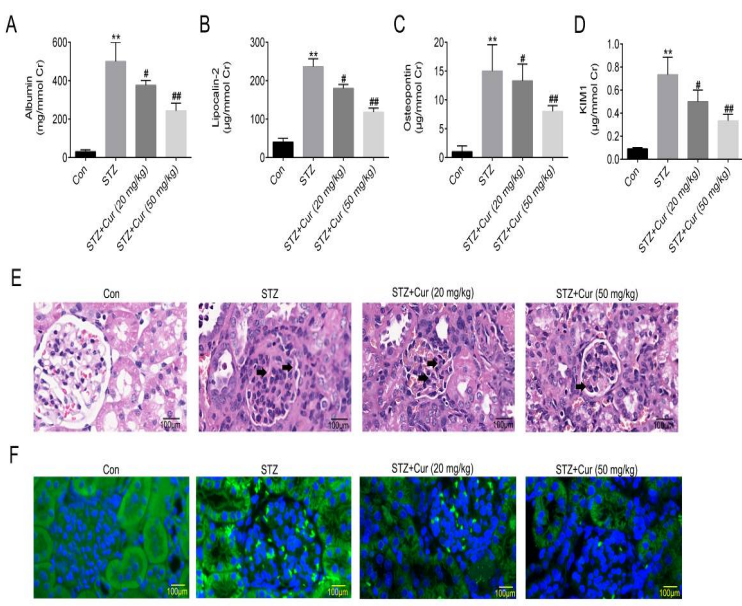

Figure 2: Effect of curcumin (Cur) treatment on alleviating kidney damage in diabetic rats. A: albumin; B: lipocalin-2; C: osteopontin; D: KIM1; $(n=10$, mean \pm SD) ${ }^{* *} p<0.01$ : STZ vs. control (Con) group, ${ }^{\#} p<$ $0.05,{ }^{\#} p<0.01$ : STZ + curcumin vs. STZ; E: haematoxylin and eosin (H\&E) staining; $F$ : TUNEL staining. STZ, streptozotocin

\section{Curcumin regulated the activity of Wnt signaling pathway in diabetic rats}

To further explore the underlying mechanism of curcumin, we determined if curcumin stimulated Wnt signaling in vivo. Whole cell lysates from tissues from the four groups of rats were prepared and examined for levels of Wnt $5 \mathrm{a}$ as well as $\beta$-catenin by using western blots and PCR (Figure $3 \mathrm{~A}$ and $\mathrm{B}$ ). The STZ group had reduced $W n t 5 a$ and $\beta$-catenin levels (by approximately $50 \%$ and $70 \%$, respectively) in the kidney tissues when compared to those of the control group; therefore, curcumin administration significantly reduced kidney damage $(p<0.05)$. In addition, high doses of curcumin showed better effects for renal restoration. Western blotting showed bands of Wnt $5 a$ and $\beta$-catenin after curcumin treatment. Furthermore, the relative protein expression levels in the STZ + Cur $(50 \mathrm{mg} / \mathrm{kg})$ group were almost the same a those of the control group (Figure $3 \mathrm{~B}$ ).

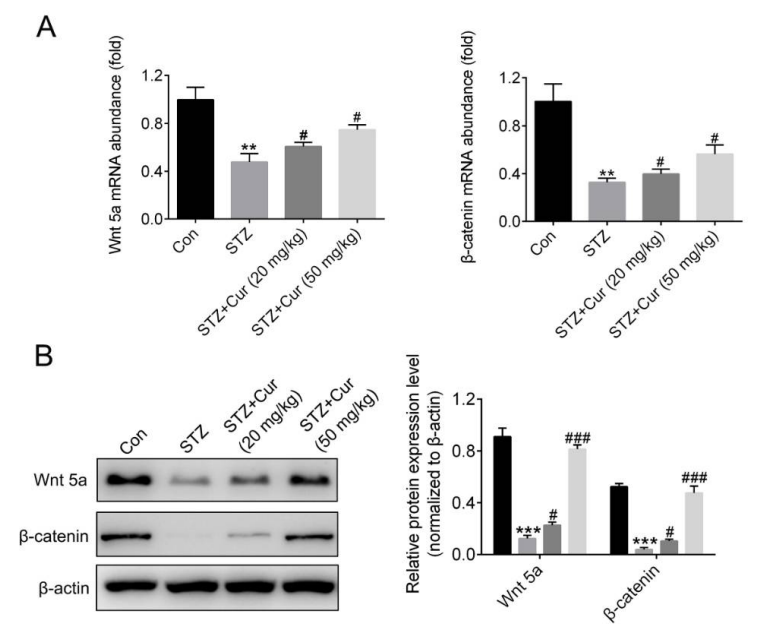

Figure 3: Effect of curcumin (Cur) treatment for regulating $W n t$ signalling. $A$ : Wnt $5 a$ and $\beta$-catenin mRNA abundance; B: Relative protein expression levels for the groups; $(\mathrm{n}=5$, mean $\pm \mathrm{SD}){ }^{\star \star} p<0.01,{ }^{* \star *} p$ $<0.001$ : STZ vs. control group, ${ }^{\#} p<0.05,{ }^{\# \#} p<0.001$ : STZ + curcumin vs. STZ. STZ, streptozotocin; Con, control

\section{Curcumin ameliorated renal damage through the Wnt signaling pathway}

We next focused on how curcumin ameliorated renal damage in diabetic rats. The staining of renal podocytes in the STZ + Cur $(50 \mathrm{mg} / \mathrm{kg})$ group showed an increased number in the diabetic glomeruli compared with that of the STZ group (Figure 4A). However, when the curcumin treatment rats were injected with XAV-939, the renal tissue staining was significantly attenuated, and the condition was the same as that of the STZ group. The apoptotic cells in the STZ + Cur $(50 \mathrm{mg} / \mathrm{kg})+$ XAV (2 mg/mL) group had increased TUNEL staining in the diabetic glomeruli (Figure 4B). These data indicated that the apoptosis-induced decrease in the diabetic glomerulus can be partially prevented by XAV939 treatment. Furthermore, the STZ + Cur (50 $\mathrm{mg} / \mathrm{kg})+\mathrm{XAV}(2 \mathrm{mg} / \mathrm{mL})$ group had decreased expression of Wnt $5 a(0.17 \pm 0.01$ normalized to $\beta$-actin) and $\beta$-catenin $(0.42 \pm 0.02$ normalized to $\beta$-actin) (Figure $4 C$ ). The mRNA abundance was about 0.5 or 0.7 -fold, respectively, compared with 
that of the STZ + Cur $(50 \mathrm{mg} / \mathrm{kg})$ group (Figure 4D).

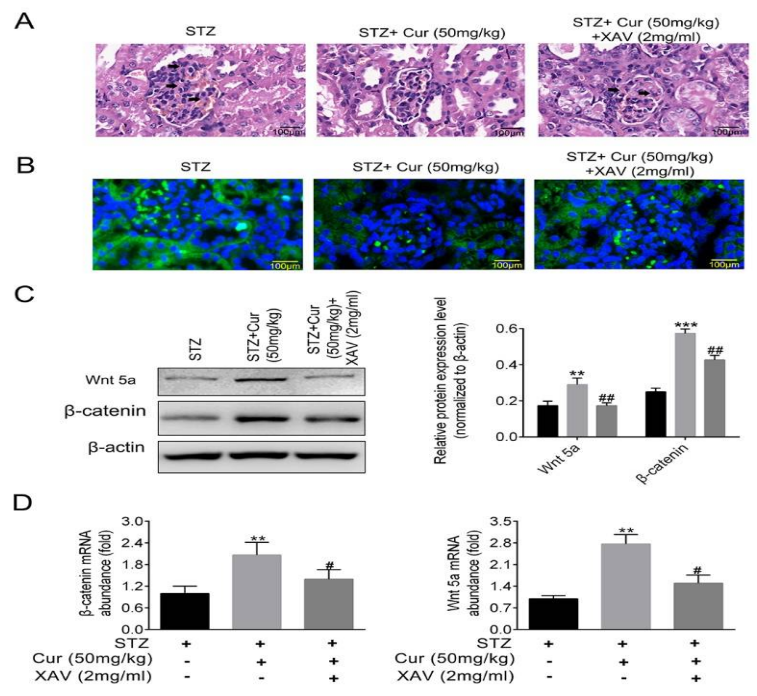

Figure 4: XAV-939 inhibited the Wnt signaling pathway. A: Haematoxylin and eosin (H\&E) staining; B: TUNEL staining $C$ : Wnt $5 a$ and $\beta$-catenin mRNA abundance; $D$ : Relative protein expression level for groups; $(\mathrm{n}=5$, mean $\pm \mathrm{SD}){ }^{\star *} p<0.01,{ }^{* * *} p<0.001$ : STZ vs. control group, ${ }^{\#} p<0.05,{ }^{\# \#} p<0.01: \mathrm{STZ}+$ Cur $(50 \mathrm{mg} / \mathrm{kg})+X A V(2 \mathrm{mg} / \mathrm{ml})$ vs. STZ + Cur $(50 \mathrm{mg} / \mathrm{kg})$. STZ, streptozotocin, Cur, curcumin

\section{DISCUSSION}

Diabetes causes damage to several target tissues, including the kidneys. Consistent with these findings, long-term oral administration of curcumin in diabetic rats has been shown to improve glucose levels [11,12]. We detected increases in body weight, MAP, and heart rate, and decreases in blood glucose and $\mathrm{HbA1c}$ after curcumin treatment. In addition, high doses of curcumin showed better effects. In diabetic nephropathy, the glomerular filtration rate increased to a hyperfiltration state. Later, interstitial proteinuria or microalbuminuria gradually appeared. Persistent proteinuria, oedema, and hypertension occurred with prolonged kidney damage. Metabolic disorder and glomerular hemodynamic changes may result from free radicals and lipid peroxidation. Curcumin treatment has antioxidant and antiinflammatory effects, and fibrotic inhibition [13].

Thus, curcumin treatment could decrease blood glucose and $\mathrm{HbA1c}$. Biological markers of renal injury can reflect changes in renal tubular damage and glomerular filtration rate. These biological markers include albumin, lipocalin-2, osteopontin, and KIM1. When the kidney is damaged, the level of albumin is high. Lipocalin2 induces renal apoptosis and osteopontin has a relationship with macrophage infiltration during renal damage. KIM1 is expressed in the apical membrane of renal proximal tubular epithelial cells after hypoxic injury. It is an early warning marker for the diagnosis of renal ischemia and hypoxic injury [14]. In the STZ group, all these proteins were significantly increased before curcumin treatment. High doses of curcumin ameliorated the renal damage and decreased these protein levels.

Glomerulus was repaired after curcumin treatment as shown by $\mathrm{H} \& \mathrm{E}$ staining, and apoptosis decreased as shown by TUNEL staining. To explore the mechanism of curcumin, western blotting and PCR were used. During kidney development, Wnt $5 \mathrm{a}$ is expressed in the kidneys and regulates the Wnt- $\beta$-catenin signaling pathway, which plays an important role in cell maturation and embryonic development $[15,16]$. Because Wnt- $\beta$-catenin promotes cell proliferation and inhibits apoptosis, it can repair cells following renal injury. Curcumin treatment increased the levels of Wnt $5 a$ and $\beta$-catenin, thereby promoting kidney damage repair $[17,18]$. Furthermore, curcumin regulated the Wnt signal pathway to improve renal damage. XAV-939 is a small molecule selective inhibitor of transcription factor $\beta$-catenin in the Wnt pathway. XAV-939 treatment $(2 \mathrm{mg} / \mathrm{mL})$ significantly reduced the protein levels of Wnt $5 a$ and $\beta$-catenin in rats administered with STZ plus Cur. The mRNA levels of Wnt $5 \mathrm{a}$ and $\beta$-catenin were also decreased by XAV-939 treatment. Collectively, curcumin reduced renal damage by modulating Wnt signaling pathway.

\section{CONCLUSION}

Curcumin alleviates renal damage in diabetic rats via modulation of Wnt signaling pathway. Thus, curcumin has the potential to be developed into a a suitable agent for the clinical management of diabetes.

\section{DECLARATIONS}

\section{Acknowledgement}

This research was supported by the innovative nurture fund of Zhongnan hospital of Wuhan University (No. znpy2017044)

\section{Conflict of Interest}

No conflict of interest associated with this work.

\section{Contribution of Authors}

We declare that this work was done by the 
authors named in this article and all liabilities pertaining to claims relating to the content of this article will be borne by the authors. Peiling Bao and Xiaoyan $\mathrm{Wu}$ designed all the experiments and revised the paper. Sai Xie and Tao $\mathrm{Li}$ performed the experiments, Aiqiao Feng wrote the paper and final approval.

\section{REFERENCES}

1. Tavafi M. Diabetic nephropathy and antioxidants. J Nephropathol 2013; 2(1): 20-27.

2. Wada J, Makino H. Inflammation and the pathogenesis of diabetic nephropathy. Clin Sci 2013; 124(3): 139-152.

3. Lenz O, Fornoni A, ljaz A, Tejada T. Role of Inflammation in Diabetic Nephropathy. Cur Diabetes Rev 2008; 4(1): 10-17.

4. Kim J, Shon E, Kim C-S, Kim JS. Renal podocyte injury in a rat model of type 2 diabetes is prevented by metformin. Exper Diabetes Res 2012; 9: e210821.

5. Prasad S, Gupta SC, Tyagi AK, Aggarwal BB. Curcumin, a component of golden spice: From bedside to bench and back. Biotechnol Adv 2014; 32(6): 1053-1064.

6. Turgut F, Bolton WK. Potential New Therapeutic Agents for Diabetic Kidney Disease. Amer J Kidney Dis 2010; 55(5): 928-940.

7. Sharma S, Kulkarni SK, Chopra K. Curcumin, the active principle of turmeric (Curcuma longa), ameliorates diabetic nephropathy in rats. Clin Exper Pharmacol Physiol 2006; 33(10): 940-945.

8. Lee FTH, Cao Z, Long DM, Panagiotopoulos S, Jerums $G$, Cooper ME, Forbes JM. Interactions between Angiotensin II and NF-KB-Dependent Pathways in Modulating Macrophage Infiltration in Experimental Diabetic Nephropathy. J Amer Soc Nephrol 2004; 15(8): 2139-2151.

9. Chiu J, Khan ZA, Farhangkhoee H, Chakrabarti S. Curcumin prevents diabetes-associated abnormalities in the kidneys by inhibiting p300 and nuclear factor-kB. Nutrition 2009; 25(9): 964-972.
10. Zhang W, Song J-K, Zhang $X$, Zhou Q-M, He G-R, XU X$N$, Rong $Y$, Zhou W-X, Du G-H. Salvianolic acid $A$ attenuates ischemia reperfusion induced rat brain damage by protecting the blood brain barrier through MMP-9 inhibition and anti-inflammation. Chin J Nat Med 2018; 16(3): 184-193.

11. He H-J, Wang G-Y, Gao $Y$, Ling $W-H$, Yu Z-W, Jin T-R. Curcumin attenuates Nrf2 signaling defect, oxidative stress in muscle and glucose intolerance in high fat dietfed mice. World J Diabetes 2012; 3(5): 94-104.

12. Brouwers $O$, Niessen PMG, Miyata T, Østergaard JA, Flyvbjerg A, Peutz-Kootstra CJ, Sieber J, Mundel PH, Brownlee $M$, Janssen BJA et al. Glyoxalase-1 overexpression reduces endothelial dysfunction and attenuates early renal impairment in a rat model of diabetes. Diabetol 2014; 57(1): 224-235.

13. Zingg J-M, Hasan ST, Meydani M. Molecular mechanisms of hypolipidemic effects of curcumin. BioFactors 2013; 39(1): 101-121.

14. Ichimura T, Bonventre JV, Bailly V, Wei H, Hession CA, Cate RL, Sanicola M. Kidney Injury Molecule-1 (KIM-1), a Putative Epithelial Cell Adhesion Molecule Containing a Novel Immunoglobulin Domain, Is Up-regulated in Renal Cells after Injury. J Biol Chem 1998; 273(7): 4135-4142.

15. Ho C, Hsu Y-C, Lei C-C, Mau S-C, Shih Y-H, Lin C-L. Curcumin Rescues Diabetic Renal Fibrosis by Targeting Superoxide-Mediated Wnt Signaling Pathways. The Amer J Med Sci 2016; 351(3): 286-295.

16. Jensen TJ, Wozniak RJ, Eblin KE, Wnek SM, Gandolfi $A J$, Futscher BW. Epigenetic mediated transcriptional activation of WNT5A participates in arsenical-associated malignant transformation. Toxicol Applied Pharmacol 2009; 235(1): 39-46.

17. He W, Dai C, Li Y, Zeng G, Monga SP, Liu Y. Wnt/3Catenin Signaling Promotes Renal Interstitial Fibrosis. J Amer Soc Nephrol 2009; 20(4): 765-776.

18. Lin C-L, Wang J-Y, Huang $Y-T$, Kuo $Y-H$, Surendran $K$, Wang F-S. Wnt/ $\beta$-Catenin Signaling Modulates Survival of High Glucose-Stressed Mesangial Cells. J Amer Soc Nephrol 2006; 17(10): 2812-2820. 